\title{
HOW VEHICULAR ENERGETIC EFFICIENCY IS INFLUENCED BY COAST DOWN COEFFICIENTS VARIATION?
}

\author{
Décio Magioli Maia ${ }^{1}$, Antonio Carlos Scardini Villela ${ }^{1}$, Rogério Nascimento de Carvalho ${ }^{1}$ \\ ${ }^{1}$ PETROBRAS
}
E-mails: deciomaia@petrobras.com.br, antonio.villela@petrobras.com.br, rogerio.carvalho@petrobras.com.br

\begin{abstract}
Energetic efficiency of Brazilian light-duty vehicles is based on urban and highway autonomies that are obtained by following NBR 7024 [1] standard, which requires that tests be carried out on chassis dynamometers. To simulate the real-world behavior of vehicles it is necessary to set the coast down coefficients in the dynamometer control system. These coefficients define the road load curve that represents wheels rolling and aerodynamic resistances to the vehicle movement, obtained at test tracks according to NBR 10312 [2] standard.

In function of environmental conditions during tests, coast down coefficients can significantly vary and impact urban and highway autonomies. This work studies the impact on urban and highway autonomies when coast down coefficients obtained on the track are varied up to $15 \%$, separately or simultaneously. Autonomy results were compared to those obtained using the original load curve, posed with the original track coefficients.
\end{abstract}

\section{INTRODUCTION}

Vehicle's urban and highway autonomies are variables that can stimulate the purchase efficient vehicles by the costumers. Therefore, the Brazilian Vehicular Labeling Program (PBEV) annually publishes those autonomies, the resulting energetic efficiency and the emissions gases from most of the vehicles sold in the national market, comparing them in each category and in overall scale.

Regarding that autonomies are declared by the vehicle manufacturers, the PBEV checks these results, through the Production Monitoring and Control (AcP). This proceeding selects, annually, some vehicles to be tested. The choice is based on the input of new technologies, better energy efficiency results than the others in the same category, low coast down coefficients and others.

One of the acceptance criteria is the maximum variation between declared and measured F0 and F2 coast down coefficients. Until two years ago it was accepted variations up to $25 \%$. Since 2015, it was reduced to $15 \%$. However, these percentages have been discussed by government regulatory offices and vehicle manufacturers.

Since there isn't a quantitative study of the F0 and F2 variation impact on urban and highway autonomies, the motivation of this paper is to investigate the maximum acceptable percentage variation for these coefficients, which keeps the autonomy results at the same level. 


\section{TEST METHODOLOGY}

In Brazil, the coast down track tests are performed according ABNT NBR 10312 [2] standard. It presents the calculation procedure to obtain the coast down load ( $\mathrm{L}_{\text {track }}$ ) as a function of vehicle speed $(\mathrm{V})$. The coast down load represents, basically, the vehicle tires rolling and air resistances. The $10 \mathrm{~km} / \mathrm{h}$ stepped track deceleration times in one $(100-30 \mathrm{~km} / \mathrm{h})$ or two $(90-70$ and $50-30 \mathrm{~km} / \mathrm{h}$ ) intervals are used to establish a second order polynomial with no first order term, as shown by eq. (1).

$L_{\text {track }}=F 0+F 2 \times V^{2}$

where F0 and F2 are the coast down track coefficients.

These coast down coefficients, F0 and F2, as well as the vehicle inertia are used to implement the coast down load curve on the chassis dynamometer, in order to allow it correctly simulate vehicle behavior on the track. From the vehicle mass, its equivalent inertia is determined based on ABNT NBR 6601 [3] standard.

In order to investigate the influence of $\mathrm{F} 0$ and $\mathrm{F} 2$ variations into the allowed $15 \%$ interval on vehicle urban and highway autonomy results, one representative Brazilian flex fuel vehicle was tested. The vehicle basic characteristics, the obtained coast down track coefficients, F0 and F2 and inertia class are displayed on table 1.

Table 1. Vehicle characteristics and coast down coefficients.

\begin{tabular}{|l|l|}
\hline Year & 2012 \\
\hline Engine & Flex fuel, 1.6 liters, port fuel injection \\
\hline Power $(\mathrm{cv})$ & $113($ ethanol $) / 110$ (gasoline) \\
\hline Mass $(\mathrm{kg})$ & 1105 \\
\hline Inertia Class $(\mathrm{kg})$ & 1247 \\
\hline F0 $(\mathrm{N})$ & 126,7 \\
\hline F2 $\left(\mathrm{N} /\left(\mathrm{km} / \mathrm{h}^{2}\right)\right)$ & 0,0381 \\
\hline
\end{tabular}

The ABNT NBR 10312 establishes that at least five pairs of opposite directions decelerations must be performed. For each run, F0 and F2 are calculated. The difference between the highest and the lowest values, for each coefficient, must not exceed $10 \%$. This percentage was also considered on F0 and F2 variation analysis.

Initially, the reference F0 and F2 values, mentioned in table 1, were diminished in $10 \%$ and $15 \%$, separately and simultaneously. The reduction of $5 \%$ is also presented, but only simultaneously, due to its low expected effects on the results. The resulting possibilities as well as their F0 and F2 modified values are presented below, on table 2:

Table 2. Coast down track coefficients sets.

\begin{tabular}{|l|l|l|}
\hline Track Coef. Set & F0 $(\mathrm{N})$ & $\mathrm{F} 2\left(\mathrm{~N} /(\mathrm{km} / \mathrm{h})^{2}\right)$ \\
\hline (F0 and F2) - REF & 126.7 & 0.0381 \\
\hline (F0 and F2)-5\% & 120.4 & 0.0362 \\
\hline (F0)-10\% & 114.0 & 0.0381 \\
\hline (F0)-15\% & 107.7 & 0.0381 \\
\hline (F0 and F2)-10\% & 114.0 & 0.0343 \\
\hline (F2)-10\% & 126.7 & 0.0343 \\
\hline (F2)-15\% & 126.7 & 0.0324 \\
\hline (F0 and F2)-15\% & 107.7 & 0.0324 \\
\hline
\end{tabular}


The coefficients reductions cause a decrease in the applied load to the vehicle by the chassis dynamometer. As load and autonomy are inversely proportional it is expected an autonomy increase. However, the magnitude of this increase is not expected to be directly proportional. This is a trial to better understand and to quantify how much the F0 and F2 variations allowed by the norms impact the vehicle autonomies. Therefore, different reduction levels and combinations were proposed.

When the track coefficients are implemented in the chassis dynamometer, the system has to compensate its own inertia, in order to impose to the vehicle only the load due to tires rolling and air resistances. After this compensation, a new load curve, called chassis dynamometer coast down load ( $\left.\mathrm{L}_{\mathrm{dyno}}\right)$ curve, is established. This new curve is a second order polynomial which contains the first order term, as presented in eq. (2).

$L_{\text {dyno }}=A++B \times V+C \times V^{2}$

where $\mathrm{A}, \mathrm{B}$ and $\mathrm{C}$ are the chassis dynamometer coast down coefficients.

The difference between $\mathrm{L}_{\text {track }}$ (eq.(1)) and $\mathrm{L}_{\text {dyno }}$ (eq.(2)) represents the dynamometer inertia, $\mathrm{L}_{\text {inertia, }}$ as shown on eq. (3). It comprises the loads due to the dynamometer mass and frictions. $L_{\text {inertia }}=L_{\text {track }}-L_{\text {dyno }}$

Those eight sets presented in table 2 were, then, implemented in the chassis dynamometer control system, which resulted in eight different load curves. The inertia was not modified.

The following step was to investigate the influence of F0 and F2 changes on the autonomies. The vehicle presented in table 1 was tested using Brazilian commercial gasoline type $\mathrm{C}$, containing $25 \% \mathrm{v} / \mathrm{v}$ of anhydrous ethanol. At least two pairs of urban and highway autonomy tests were performed, regarding each one of the eight possibilities proposed above. The tests followed the ABNT NBR 7024 [1] standard and the autonomies were calculated based on the carbon balance method. The emissions gases, necessary to the carbon balance calculations, were measured following ABNT NBR 6601 [3].

\section{RESULTS}

Figure 1 shows F0 and F2 separated effects on urban autonomy. It illustrates the reference (F0 and $\mathrm{F} 2),(\mathrm{F} 0-10 \%),(\mathrm{F} 0-15 \%),(\mathrm{F} 2-10 \%)$ and $(\mathrm{F} 2-15 \%)$ sets autonomy values, in $\mathrm{km} / \mathrm{L}$, and the percent difference among the reduced ones to the reference.

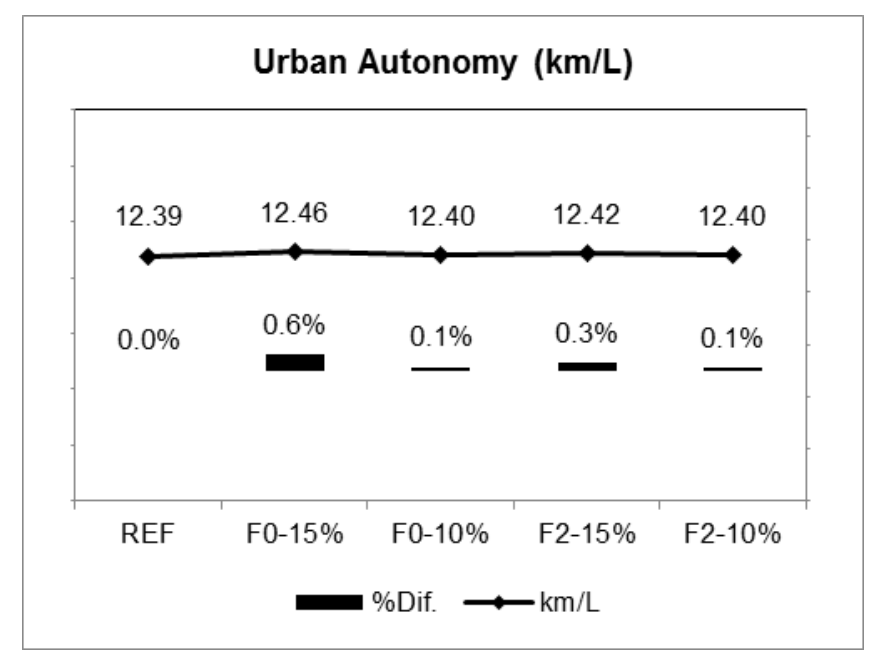

Figure 1. Urban autonomy results for F0 and F2 separated reductions.

It can be noticed that urban autonomy results were very similar when isolated reductions of F0 and F2 were imposed to the load curves. The maximum percent difference related to the 
reference values was $0.6 \%$, which is lower than $1.8 \%$, the estimated autonomy results uncertainty of the test laboratory.

The F0 reduction means a constant subtraction, in Newtons, from the reference load curve. Regarding that load curve is a second order polynomial, F0 reduction is more significant at lower speeds than at higher speeds (figure 2). However, the demanded power at lower speeds is also low. It could minimize autonomy differentiation on these points due to F0 reductions.

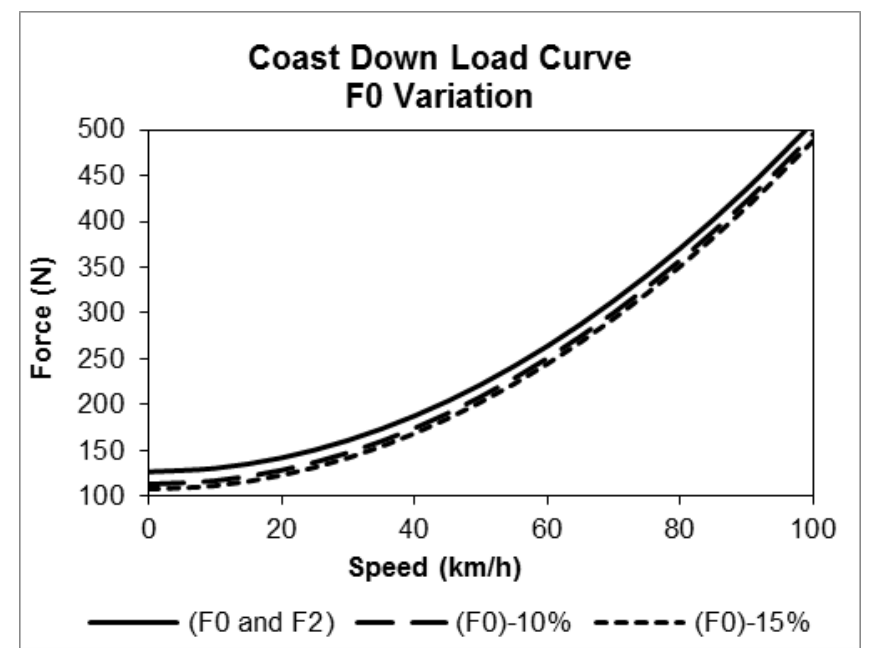

Figure 2. F0 variation effect on the coast down load curve.

On the other hand, F2 reductions affect the load curve more significantly at higher speeds (figure 3). Nevertheless, the urban driving cycle FTP-75, adopted by ABNT NBR 7024 [1], do not reach such speeds during significant time. Despite it reaches $90 \mathrm{~km} / \mathrm{h}$ at maximum, its mean speed is around $33 \mathrm{~km} / \mathrm{h}$. Therefore, the urban autonomy was not affected by the F2 reductions of $10 \%$ and $15 \%$.

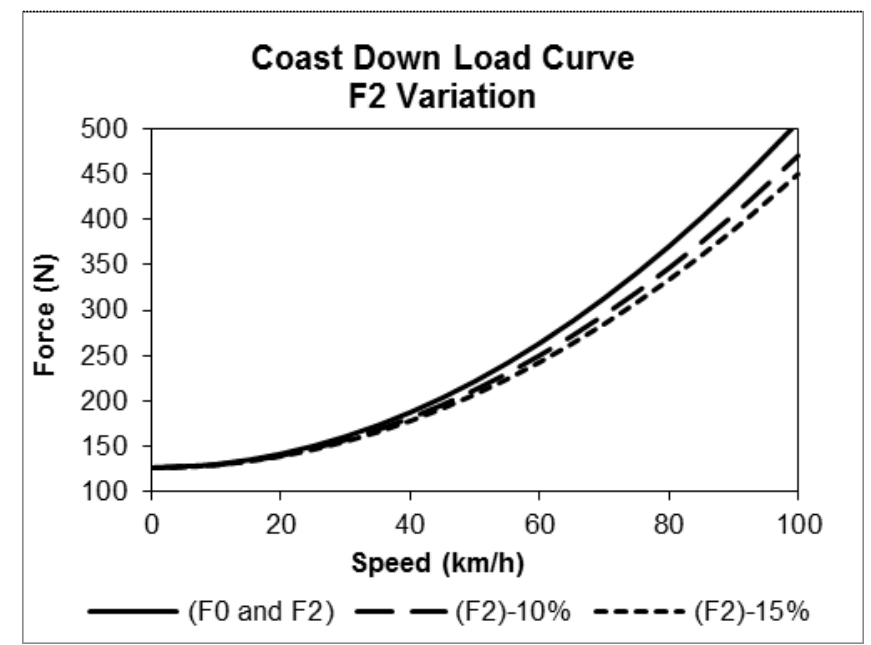

Figure 3. F2 variation effect on the coast down load curve. 
Figure 4 presents the effects F0 and F2 separated reductions on highway autonomy. It follows the same format applied to figure 1.

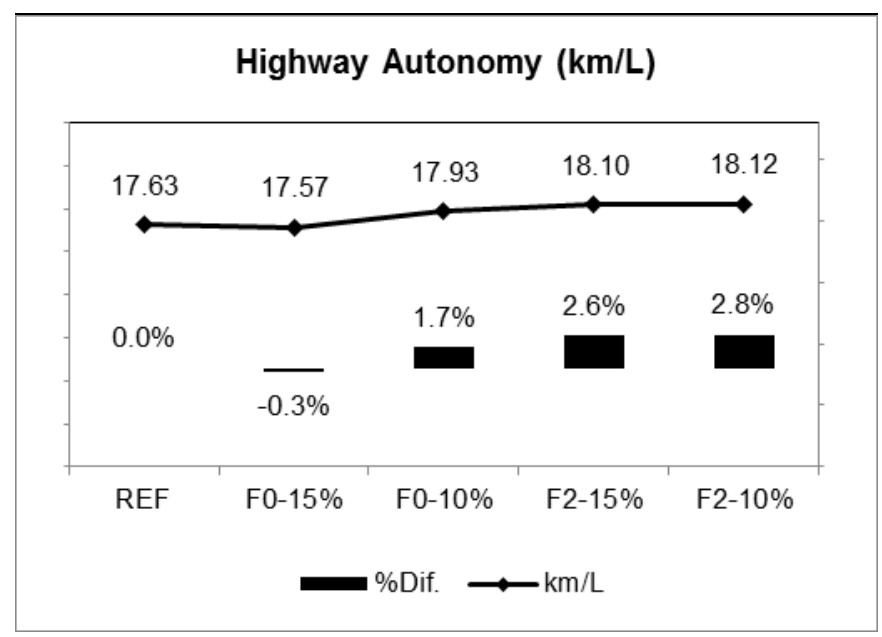

Figure 4. Highway autonomy results for F0 and F2 separated reductions.

F0 reductions also resulted in similar autonomy values for the highway case. The percent differences to the reference are located into the test uncertainty range $(1.8 \%)$. However, the $1.7 \%$ obtained for $\mathrm{F} 0-10 \%$ condition is a boundary value, which indicates that F0 isolated influence cannot be totally neglected. In general, the low F0 influence occurred because the highway driving cycle (adopted by ABNT NBR 7024 [1]) is performed, mostly, at high speeds (mean speed $=77 \mathrm{~km} / \mathrm{h}$ ), where F0 constant subtraction is small compared to the high speed load values (figure 2).

However, when F2 was reduced the autonomy values increased $2.6 \%$ and $2.8 \%$ compared to the reference, beyond the $1.8 \%$ test laboratory uncertainty and, therefore, resulting in highway autonomy increase. It can be explained by the higher influence of F2 on the load curve at high speeds, typical of highway driving cycle (figure 3 ).

Figure 5 presents simultaneous reduction of $\mathrm{F} 0$ and $\mathrm{F} 2$. It illustrates the reference (F0 and F2), (F0and F2-5\%), (F0 and F2-10\%) and (F0 and F2-15\%) sets autonomy values, in $\mathrm{km} / \mathrm{L}$, and the percent difference among the reduced ones to the reference.

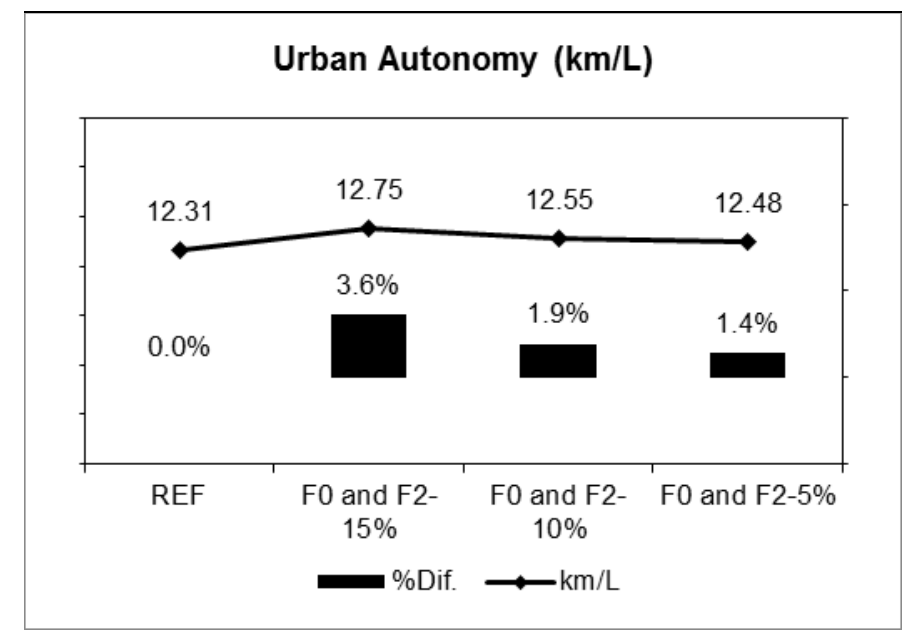

Figure 5. Urban autonomy results for F0 and F2 simultaneous reductions.

It is possible to observe that F0 and F2 simultaneous reductions affected the urban autonomy at higher levels compared to the separated effects. With a 5\% coefficients simultaneous reduction, it was found a $1.4 \%$ autonomy increase. This percent difference is located into the 
test laboratory uncertainty, but it indicates a trend that was confirmed when the coefficients reductions went further on $10 \%$ and $15 \%$. For these F0 and F2 simultaneous reductions, the urban autonomy values were improved in $1.9 \%$ and $3.6 \%$ compared to the reference, respectively.

The urban autonomy increased progressively as both coefficients were reduced. This behavior is coherent with the variations verified for the load curves, which decreased progressively with F0 and F2 reductions (figure 6).

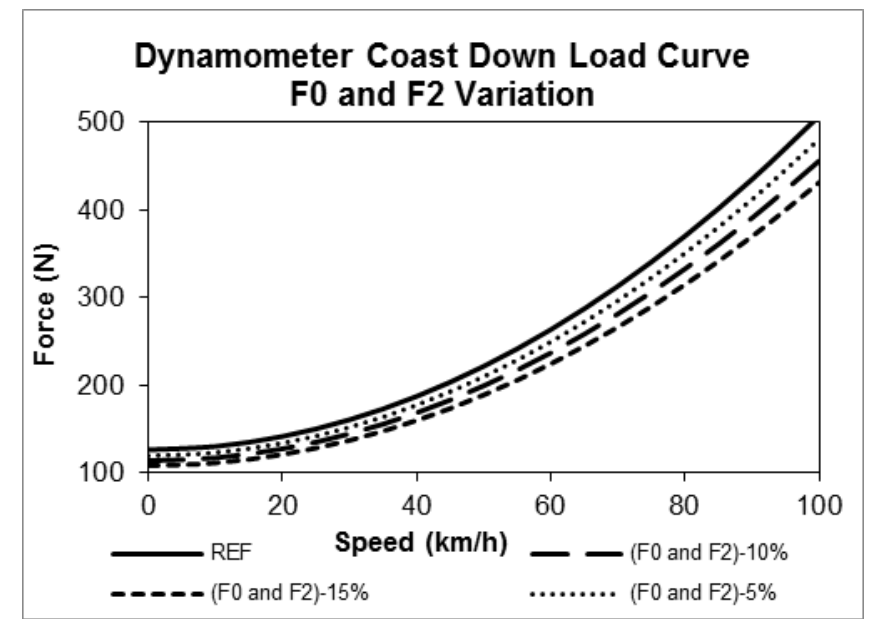

Figure 6. F0 and F2 variation effect on the coast down load curve.

Figure 7 presents F0 and F2 simultaneous reductions effects on the highway autonomy. It follows the same format applied to figure 5.

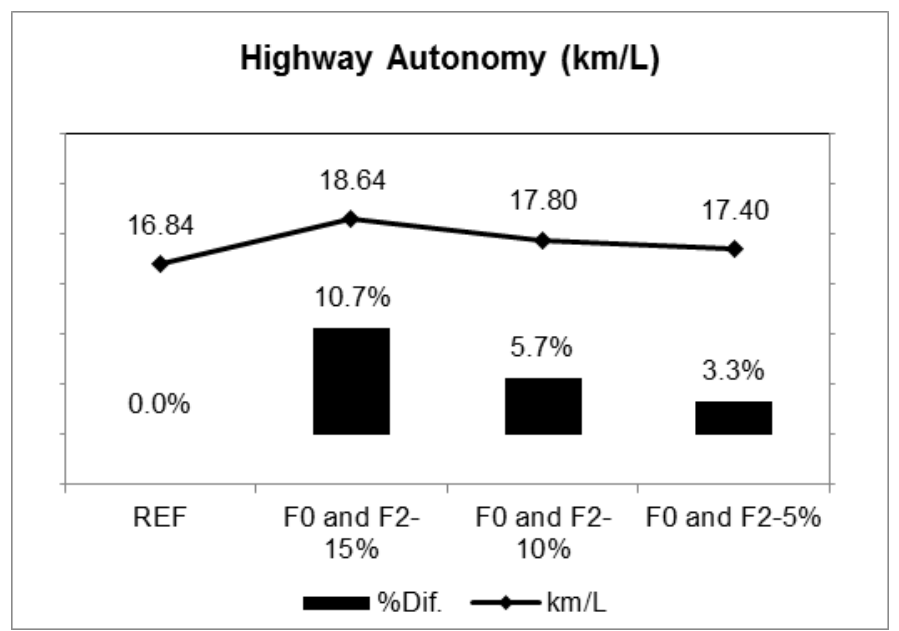

Figure 7. Highway autonomy results for F0 and F2 simultaneous reductions.

It can be noticed the highway autonomy also increased progressively as F0 and F2 were reduced. However, the increase magnitude was greater than that observed for the urban case. It started from $3.3 \%$ with $5 \%$ coefficients reduction up to $10,7 \%$ with $15 \%$ reduction. This higher increase in the highway case can be explained by the greater differences among the load curves at high speeds, typical from highway driving cycle (figure 6). In this situation the demanded power is also high, which can highlight the impact of the load changes on the autonomy. 


\section{SUMMARY/CONCLUSIONS}

From the obtained data, it can be verified that the urban autonomy was not affected by F0 and F2 separated reductions. The results percent differences to the reference autonomies values were lower than the test uncertainty, which is $1.8 \%$.

Although the highway autonomy was not affected by the F0 coefficient reduction, it was affected by the F2 coefficient $10 \%$ reduction, generating a $2.8 \%$ increase in the autonomy result.

The F0 and F2 coefficients simultaneous reduction of $15 \%$ increased urban autonomy in $3.6 \%$, while $10 \%$ and $5 \% \mathrm{~F} 0$ and $\mathrm{F} 2$ reductions did not affect autonomy results. Regarding the highway case, the proposed simultaneous coefficients reductions of $15 \%, 10 \%$ and $5 \%$, caused autonomies increase of $10,7 \%, 5,7 \%$ and $3.3 \%$.

Summarizing, there was no influence on autonomy when F0 coefficient was reduced. F2 reduction affected only the highway autonomy, increasing it. The reduction of both coefficients increased highway autonomy and also urban, but in lower percentages.

In order to establish F0 and F2 coefficients variation limits, based on the impact on the autonomy results, further tests should be carried out. These tests should consider vehicles size, varying from micro compacts to larges, since the mass may influence the final results.

\section{REFERENCES}

1. ABNT Brazilian Norm, "Light road vehicles - Fuel consumption determination - Test method", ABNT NBR 7024, Rev. Apr. 2010.

2. ABNT Brazilian Norm, "Light duty road vehicles - Road load measurement and dynamometer simulation using coast down techniques", ABNT NBR 10312, Rev. Sep. 2014.

3. ABNT Brazilian Norm, "Light road vehicles - Determination of hydrocarbon, carbon monoxide, nitrogen oxide, carbon dioxide and particulate material on exhaust gas", ABNT NBR 6601, Rev. Oct. 2012.

\section{CONTACT INFORMATION}

Decio Magioli Maia: e-mail: deciomaia@ petrobras.com.br

Cellular: +55-21-981215461 